\title{
Inventory of black hole binaries
}

\author{
Jerome A. Orosz ${ }^{1}$ \\ Sterrekundig Instituut, Universiteit Utrecht, \\ Princetonplein 5, NL-3584CC Utrecht, Nederland
}

\begin{abstract}
A small group of X-ray binaries currently provides the best evidence for the existence of stellar-mass black holes. These objects are interacting binary systems where the X-rays arise from accretion of material onto a compact object (i.e., an object with a radius of less than a few hundred $\mathrm{km}$ ). In some favourable cases, optical studies of the companion star lead to dynamical mass estimates for both components. In 17 cases, the mass of the compact object in an X-ray binary has been shown to exceed the maximum mass of a stable neutron star (about $3 \mathrm{M}_{\odot}$ ), which leads to the conclusion that these objects are black holes. In this contribution I will review the basic properties of these black hole binaries.
\end{abstract}

\section{Definition and source selection}

Black holes represent an extreme manifestation of Einstein's theory of general relativity. As an observational astronomer, I will not consider any of the detailed theory of black holes but will instead consider only the practical question of how to find them. The usual route is by the process of elimination. In the current universe, black holes must form via the gravitational collapse of a dying star. Three outcomes are possible, depending on the mass of the degenerate core: a white dwarf, a neutron star, or a black hole. The mass of a white dwarf cannot exceed the well-known Chandrasekhar limit, and a neutron star has a somewhat analogous upper mass limit, generally thought to be on the order of $3 \mathrm{M}_{\odot} \odot$ (Rhoades \& Ruffini 1974; Kalogera \& Baym 1996). Once the mass of the degenerate core exceeds $\sim 3 \mathrm{M}_{\odot}$, no known force can halt the gravitational collapse, and a black hole must be formed. Given this, we have a relatively straightforward observational definition of a black hole: $A$ black hole is a compact object with a mass greater than three solar masses, where 'compact' in this context means an object with a radius smaller than about $100 \mathrm{~km}$ (i.e., much less than the radius of normal stars).

Since black holes are dark, the only way one could hope to observe them is through their gravitational influence on surrounding matter. Early attempts to search catalogs of spectroscopic binaries to look for single-lined binaries with massive and undetected companions were not successful (Zel'dovich \& Guseynov 1966; Trimble \& Thorne 1969). A far more efficient approach for source selection has its roots in the 1960s when Zel'dovich and others realized that if a black

\footnotetext{
${ }^{1}$ Present address: Department of Astronomy, San Diego State University, 5500 Campanile Drive, San Diego, CA 92182-1221, USA
} 
hole accreted material (either from a nearby companion star or the interstellar medium), then it might shine brightly in X-rays and $\gamma$ rays. Today we know of a few hundred bright $\mathrm{X}$-ray sources which must be powered by accretion onto a compact object, either a neutron star or a black hole (i.e., rapid variability in many cases indicates a size scale of a few hundred $\mathrm{km}$ or less). All of the black hole candidates discussed below were selected on the basis of their X-ray activity, although two of the sources, V404 Cyg and V4641 Sgr, were previously known (optically) variable stars that were essentially ignored until they were associated with bright X-ray sources. Thus, one should keep in mind that owing to special circumstances needed to observe them (i.e., interacting binaries), the list of presently known stellar-mass black holes is probably a very biased sample and may not be representative of the general population of black holes.

\section{Mass limits}

By my definition above, to establish the presence of a black hole in a given system one must show that there is a compact object with a mass greater than $3 \mathrm{M}_{\odot}$. Establishing a lower limit to the mass is relatively easy since in a singlelined spectroscopic binary, the observed radial velocity curve of the visible star can be used to set a firm lower limit on the mass of the unseen companion:

$$
f(M) \equiv \frac{P K_{2}^{3}}{2 \pi \mathrm{G}}=\frac{M_{\mathrm{BH}}^{3} \sin ^{3} i}{\left(M_{\mathrm{BH}}+M_{2}\right)^{2}},
$$

where $P$ is the binary orbital period, $K_{2}$ is the semiamplitude of the companion's radial velocity curve, $\mathrm{G}$ is the gravitation constant, $i$ is the inclination of the orbit to the line of sight ( $i=90^{\circ}$ for an orbit seen edge-on), and where $M_{\mathrm{BH}}$ and $M_{2}$ are the masses of the invisible object and the companion star, respectively. One can easily see that $f(M)$ (generally called the 'optical mass function' or 'mass function') is always less than $M_{\mathrm{BH}}$ since $M_{2}>0$ and $\sin i \leq 1$ always. Thus a firm lower limit on the mass of the compact object can be obtained from two relatively simple measurements.

In real life, of course, things are always a bit more complicated. For starters, in many 'cases one cannot even see spectral lines from the companion star in many of the persistently X-ray bright sources. Needless to say this precludes any attempts at dynamical mass estimates. In other cases, the companion star may not be uniformly bright over its surface owing to heating by the X-ray source. If so, then the 'centre of light' will not be the same as the 'centre of mass' and the observed radial velocity curve will be different than the true one. Since the value of the mass function depends on $K_{2}^{3}$, small changes in $K_{2}$ can lead to relatively large changes in $f(M)$. Fortunately, many of the potential difficulties related to X-ray heating are largely absent in the subset of the Xray binaries known as ' $\mathrm{X}$-ray transients'. As the name suggests, the $\mathrm{X}$-ray luminosity in these sources is highly variable (dynamic ranges of $10^{6}$ to $10^{7}$ are not uncommon). Dynamical observations of these systems are generally reliable, provided one observes the system after the $X$-ray activity has completely ceased (see Phillips, Shahbaz \& Podsiadlowski 1999; Shahbaz et al. 1999a; and Shahbaz et al. 2000 for discussions regarding X-ray heating in GRO J1655-40).

Table 1 lists the mass functions for 17 systems that are widely believed to contain black holes. Fourteen of these systems are transient X-ray sources 
Table 1. Basic system parameters for the fourteen transient and three persistent black hole binaries.

\begin{tabular}{|c|c|c|c|c|c|c|c|}
\hline source & $\begin{array}{l}P \\
\text { (d) }\end{array}$ & $\begin{array}{l}f(M) \\
\left(M_{\odot}\right)\end{array}$ & $\begin{array}{l}v_{\text {rot }} \sin i \\
\left(\mathrm{~km} \mathrm{~s}^{-1}\right)\end{array}$ & $\stackrel{i}{(\operatorname{deg})}$ & $\stackrel{q}{\left(M_{\mathrm{BH}} / M_{2}\right)}$ & $\begin{array}{l}M_{\mathrm{BH}} \\
\left(\mathrm{M}_{\odot}\right)\end{array}$ & refs. \\
\hline GRO J $0422+32$ & $0.2121600(2)$ & $1.19 \pm 0.02$ & $90_{-27}^{+22}$ & $44 \pm 2$ & $3.2-13.2$ & $3.66-4.97$ & $1-3$ \\
\hline $\begin{array}{l}\text { A } 0620-00 \\
\text { GRS } 1009-45 \\
\text { XTE J } 1118+480 \\
\text { GS } 1124-683 \\
\text { 4U 1543-47 } \\
\text { XTE J } 1550-564 \\
\text { GRO J } 1655-40 \\
\text { H 1705-250 } \\
\text { SAX J } 1819.3-2525 \\
\text { XTE J } 1859+226 \\
\text { GRS } 1915+105 \\
\text { GS 2000+25 } \\
\text { GS } 2023+338\end{array}$ & $\begin{array}{l}0.3230160(5) \\
0.285206(2) \\
0.169930(4) \\
0.432606(3) \\
1.116407(3) \\
1.5435(5) \\
2.6219(2) \\
0.521(1) \\
2.81730(1) \\
0.382(3) \\
34(2) \\
0.3440915(9) \\
6.4714(1)\end{array}$ & $\begin{array}{l}2.72 \pm 0.06 \\
3.17 \pm 0.12 \\
6.1 \pm 0.3 \\
3.01 \pm 0.15 \\
0.25 \pm 0.01 \\
6.86 \pm 0.71 \\
2.73 \pm 0.09 \\
4.86 \pm 0.13 \\
3.13 \pm 0.13 \\
7.4 \pm 1.1 \\
9.5 \pm 3.0 \\
5.01 \pm 0.12 \\
6.08 \pm 0.06\end{array}$ & $\begin{array}{l}83 \pm 5 \\
\dddot{114} \pm 4 \\
106 \pm 13 \\
46 \pm 2 \\
90 \pm 10 ? \\
93 \pm 3 \\
<79 \\
98.9 \pm 1.5 \\
\cdots \\
\dddot{86} \pm 8 \\
38.8 \pm 1.1\end{array}$ & $\begin{array}{l}40.8 \pm 3.0 \\
67 ? \\
81 \pm 2 \\
54 \pm 2 \\
20.7 \pm 1.5 \\
72 \pm 5 \\
70.2 \pm 1.2 \\
760 \\
75 \pm 2 \\
70 \pm 2 ? \\
70 \pm 0 \pm 1.3 \\
56 \pm 4\end{array}$ & $\begin{array}{l}13.3-18.3 \\
6.3-8.0 ? \\
22.7-28.8 \\
4.8-8.8 \\
3.2-4.0 \\
>12 \\
2.4-2.7 \\
>18.9 \\
2.22-2.39 \\
\cdots \\
\dddot{1} .9-28.9 \\
16.1-18.9\end{array}$ & $\begin{array}{l}8.70-12.86 \\
3.64-4.747 \\
6.48-7.19 \\
6.47-8.18 \\
8.45-10.39 \\
8.36-10.76 \\
6.03-6.57 \\
5.64-8.30 \\
6.82-7.42 \\
7.6-12.0 ? \\
10.0-18.0 ? \\
7.15-7.78 \\
10.06-13.38\end{array}$ & $\begin{array}{l}4-6 \\
7,8 \\
9-11 \\
12-15 \\
16 \\
17 \\
18-20 \\
21-23 \\
24,25 \\
26 \\
27-29 \\
30-32 \\
33,34\end{array}$ \\
\hline $\begin{array}{l}\text { LMC X-3 } \\
\text { LMCX-1 } \\
\text { Cyg X-1 }\end{array}$ & $\begin{array}{l}1.70479(4) \\
4.2288(6) \\
5.59983(2)\end{array}$ & $\begin{array}{l}2.29 \pm 0.32 \\
0.14 \pm 0.05 \\
0.244 \pm 0.005\end{array}$ & $\begin{array}{l}130 \pm 20 \\
\dddot{94} \pm 5\end{array}$ & $\begin{array}{l}67 \pm 3 \\
\sim 63 ? \\
35 \pm 5\end{array}$ & $\begin{array}{l}1.1-2.0 \\
0.3-0.7 ? \\
0.50-0.57\end{array}$ & $\begin{array}{l}5.94-9.17 \\
4.0-10.0 ? \\
6.85-13.25\end{array}$ & $\begin{array}{l}35-37 \\
38 \\
39-42\end{array}$ \\
\hline
\end{tabular}

References: 1: Webb et al. 2000; 2: Harlaftis et al. 1999; 3: Gelino \& Harrison 2002; 4: Leibowitz, Hemar \& Orio 1998; 5: Marsh, Robinson \& Wood 1994; 6: Gelino, Harrison \& Orosz 2001; 7: Filippenko et al. 1999; 8: Gelino 2002; 9: Wagner et al. 2001; 10: Orosz 2001; 11: McClintock et al. 2001; 12: Orosz et al. 1996; 13: Casares et al. 1997; 14: Shahbaz, Naylor \& Charles 1997; 15: Gelino, Harrison \& McNamara 2001; 16: Orosz et al. 2002b; 17: Orosz et al. 2002a; 18: Greene, Bailyn \& Orosz 2001; 19: Shahbaz et al. 1999a; 20: Israelian et al. 1999; 21: Remillard et al. 1996; 22: Filippenko et al. 1997; 23: Harlaftis et al. 1997; 24: Orosz et al. 2001; 25: Orosz et al. 2002c; 26: Filippenko \& Chornock 2001; 27: Greiner, Cuby \& McCaughrean 2001; 28: Mirabel \& Rodriguez 1994; 29: Fender et al. 1999; 30: Chevalier \& Ilovaisky 1993; 31: Harlaftis, Horne \& Filippenko 1996; 32: Gelino 2001; 33: Casares \& Charles 1994; 34: Shahbaz et al. 1994; 35: van der Klis et al. 1985; 36: Cowley et al. 1983; 37: Kuiper, van Paradijs \& van der Klis 1988; 38: Hutchings et al. 1987; 39: Brocksopp et al. 1999; 40: Ninkov, Walker \& Yang 1987; 41: Gies \& Bolton 1986; 42: Herrero et al. 1995.

with relatively low-mass companions $\left(M_{2} \lesssim 1 \mathrm{M}_{\odot}\right.$ generally), and the remaining three systems have massive $\mathrm{O} / \mathrm{B}$ companions whose bolometric luminosity is comparable to or exceeds the X-ray luminosity of the accreting object. Six of the systems have mass functions which are above $\sim 5 \mathrm{M}_{\odot}$, and six more have mass functions more than $\sim 3 \mathrm{M}_{\odot}$. Based on the mass functions alone, we can safely conclude these systems contain black holes since the compact objects in them are too massive to be neutron stars. Although the other five systems have relatively small mass functions, we can be reasonably sure these systems also contain black holes since the currently best estimates for the compact object masses (see the next section) are all above $\sim 3.5 \mathrm{M}_{\odot}$. In addition to the sources listed in Table 1, there are a few dozen sources which are believed to contain a black hole on the basis of their X-ray spectra and temporal properties (e.g., Tanaka \& Lewin 1995; Liu, van Paradijs \& van den Heuvel 2001).

\section{Towards actual masses}

If possible, we want to do better than simply establishing a lower limit to the mass of a black hole. If we think of the companion star as a test particle orbiting the black hole, then clearly in order to measure the actual mass of the black hole after its mass function has been measured, one must be able to convert projected radial velocities into actual radial velocities (i.e., the inclination $i$ is needed) and to find out how far the star is from the binary centre-of-mass (i.e., one needs to know the mass ratio or the mass of the companion star).

The procedure to derive astrophysically interesting parameters of a binary system is conceptually simple. One can make a model of a close binary system 
that is specified by several parameters such as the orbital period, the separation between the stars, the mass ratio, the relative radii of the stars, the temperatures of the stars, the inclination to the line of sight, etc. Then, given this model, one can compute various observable properties of this binary such as the radial velocity curves, the light curves in various bandpasses, eclipse duration(s) if any, the rotational velocities of the stars, etc. The goal then is to find a set of input model parameters that can best match the set of observed properties of the binary of interest. Given the optimal input parameters, one can then compute astrophysically interesting quantities such as the masses of the components.

Models of close binary stars are relatively easy to construct, and have been used for over thirty years (e.g., Wilson \& Devinney 1971; Lyutyi, Syunyaev, Cherepashchuk 1973; Hutchings 1974; Avni \& Bahcall 1975). The difficult part has been related to the inverse problem of parameter optimisation since the parameter space to search is usually vast and often parameters can be tightly correlated. Common ways to tackle the inverse problem include 'differential corrections' and related techniques (e.g., Wilson \& Devinney 1971) and systematic 'grid searches' (e.g., Kuiper, van Paradijs \& van der Klis 1988). More recently, I have been using optimisation techniques based on genetic algorithms (Orosz et al. 2002a). Genetic algorithms are very robust, and can allow for accurate estimation of uncertainties on the fitted and derived parameters.

In the case of the black hole binaries, the main observables usually are a radial velocity curve for the companion star (this basically sets the scale of the binary), a light curve for the companion in one or more bandpasses (these mainly constrain the inclination), and in most cases a measurement of the mean projected rotational velocity of the companion star (this sets the mass ratio). In nearly cases (with the possible exception of SAXJ1819.3-2525), the X-ray source is not eclipsed, and one can set an upper limit to the inclination. Given these observations, it is not an unduly difficult problem (in principle!) to find a good binary model for most of the black hole systems listed in Table 1.

However, as before, in real life things are more complicated. In many cases, the observed light curve is not entirely due to the companion star, owing to the presence of extra sources of light (usually from an accretion disk). In the optical, the mean light curve can change from one year to the next (e.g., Orosz et al. 1996; Leibowitz, Hemar \& Orio 1998; Pavlenko et al. 2001). Presumably these long-term changes are related to the accretion disk. From spectroscopy, one can estimate the light attributable to the accretion disk (e.g., Marsh, Robinson \& Wood 1994) and correct for it in the models. Alternatively, it is generally assumed that the contamination from the accretion disk is small in the infrared $J$. $H$, and $K$ bands (e.g., Shahbaz et al. 1994; Gelino, Harrison \& McNamara 2001). There have been a few attempts to directly measure the disk contamination in the infrared with spectroscopy, but the results so far are not terribly constraining (Shahbaz et al. 1996; Shahbaz, Bandyopadhyay \& Charles 1999b).

I have summarised in Table 1 the best (in my opinion) measurements of the rotational velocities and inclinations for the 17 black hole binaries. Relatively uncertain values are denoted by '?'. In some cases there is reasonably good agreement between inclination measurements made by different groups (e.g., GS 1124-683), while in other cases there have been a wide range of measurements (e.g., Cyg X-1, GRO J0422+32). I have computed $\sim 1 \sigma$ ranges for the black 


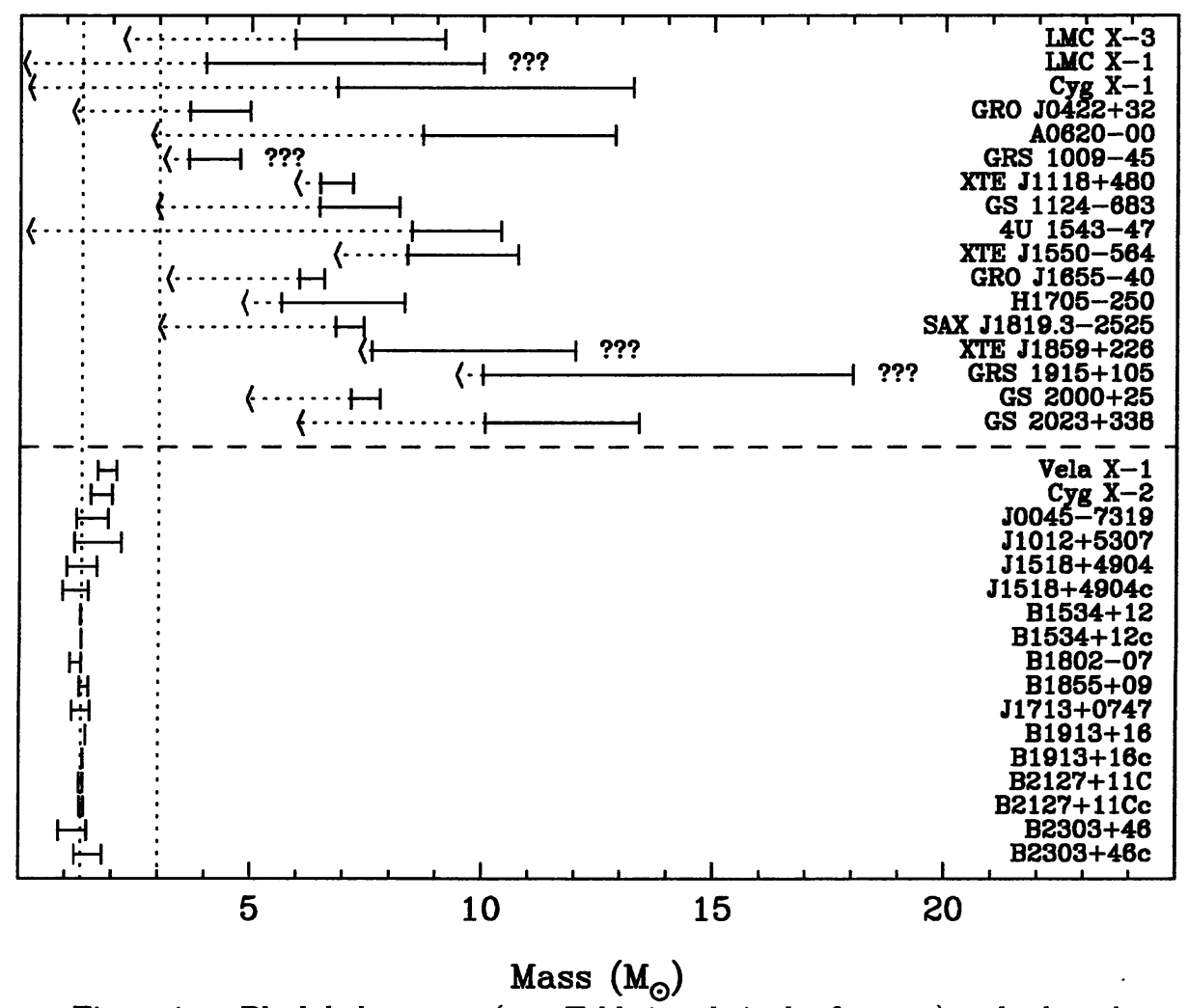

Figure 1. Black hole masses (top, Table 1 and cited references) and selected neutron star masses (bottom, Thorsett \& Chakrabarty 1999). Ranges are $1 \sigma$ in most cases. Arrows in the top indicate measured mass functions.

hole mass and mass ratio using a simple Monte Carlo code that makes use of numerical Roche lobe integrations to map a rotational velocity into a mass ratio. In some cases, rotational velocities are not available and instead I have guessed the mass of the companion in order to arrive at a mass range for the black hole. Figure 1 shows graphically the black hole mass ranges and, for comparison, masses for neutron stars. All things considered, many of the masses are known reasonably well, but one should always keep in mind potential systematic errors such as those related to unstable light curves, etc.

\section{References}

Avni, Y., Bahcall, J.N. 1975, ApJ 197, 675

Brocksopp, C., Tarasov, A.E., Lyutyi, V.M., Roche, P. 1999, A\&A 343, 86

Casares, J., Charles, P. A. 1994, MNRAS (Letters) 271, L5

Casares, J., Martin, E.L., Charles, P.A., et al. 1997, New Astron. 1, 299

Chevalier, C., Ilovaisky, S.A. 1993, A\&A 269, 301

Cowley, A.P., Crampton, D., Hutchings, J.B., et al. 1983, ApJ 272, 118

Fender, R.P., Garrington, S.T., McKay, D.J., et al. 1999, MNRAS 304, 865 
Filippenko, A.V., Matheson, T., Leonard, D.C., et al. 1997, PASP 109, 461

Filippenko, A.V., Leonard, D.C., Matheson, T., et al. 1999, PASP 111, 969

Filippenko, A.V., Chornock, R. 2001, IAU Circular No. 7644

Gelino, D.M., Harrison, T.E., McNamara, B.J. 2001, AJ 122, 971

Gelino, D.M., Harrison, T.E., Orosz, J.A. 2001, AJ 122, 2668

Gelino, D.M. 2001, PhD thesis, New Mexico State University, USA

Gelino, D.M. 2002, BAAS 200, \#08.11

Gelino, D.M., Harrison, T.E. 2002, ApJ in press

Gies, D.R., Bolton, C.T. 1986, ApJ 304, 371

Greene, J., Bailyn, C.D., Orosz, J.A. 2001, ApJ 554, 1290

Greiner, J., Cuby, J.G., McCaughrean, M.J. 2001, Nature 414, 522

Harlaftis, E.T., Horne, K., Filippenko, A.V. 1996, PASP 108, 762

Harlaftis, E., Steeghs, D., Horne, K., Filippenko, A.V. 1997, AJ 114, 1170

Harlaftis, E., Collier, S., Horne, K., Filippenko, A.V. 1999, A\&A 341, 491

Herrero, A., Kudritzki, R.-P., Gabler, R., et al. 1995, A\&A 297, 556

Hutchings, J.B. 1974, ApJ (Letters) 193, L61

Hutchings, J.B., Crampton, D., Cowley, A.P., et al. 1987, AJ 94, 340

Israelian, G., Rebolo, R., Basri, G., Casares, J., Martin, E.L. 1999, Nature 401, 142

Kalogera, V., Baym, G. 1996, ApJ (Letters) 470, L61

Kuiper, L., van Paradijs, J., van der Klis, M. 1988, A\&A 203, 79

Leibowitz, E.M., Hemar, S., Orio, M. 1998, MNRAS 300, 463

Liu, Q.Z., van Paradijs, J., van den Heuvel, E.P.J. 2001, A\&A 368, 1021

Lyutyi, V.M., Syunyaev, R.A., Cherepashchuk, A.M. 1973, Astron. Zh. 50, 3 (= Soviet Astron. 17, 1)

Marsh, T.R., Robinson, E.L., Wood, J.H. 1994, MNRAS 266, 137

McClintock, J.E., Garcia, M.R., Caldwell, M., et al. 2001, ApJ (Letters) 551, L147

Mirabel, I.F., Rodriguez, L.F. 1994, Nature 371, 46

Ninkov, Z., Walker, G.A.H., Yang, S. 1987, ApJ 321, 425

Orosz, J.A. 2001, ATel \#67

Orosz, J.A., Bailyn, C.D., McClintock, J.E., Remillard, R.A. 1996, ApJ 468, 380

Orosz, J.A., Kuulkers, E., van der Klis, M., McClintock, J.E., Garcia, M.R., Callanan, P.J., Bailyn, C.D., Jain, R.K., Remillard, R.A. 2001, ApJ 555, 489

Orosz, J.A., Groot, P.J., van der Klis, M., McClintock, J.E., Garcia, M.R., Zhao, P., Jain, R.K., Bailyn, C.D., Remillard, R.A. 2002a, ApJ 568, 845

Orosz, J.A., et al. $2002 \mathrm{~b}$, in preparation

Orosz, J.A., et al. 2002c, in preparation

Pavlenko, E.P., Kuznetsova, Y.G., Shugarov, S.Yu., Petrov, V.S. 2001, Ap\&SS Suppl. 276,65

Phillips, S.N., Shahbaz, T., Podsiadlowski, Ph. 1999, MNRAS 304, 839

Remillard, R.A., Orosz, J.A., McClintock, J.E., Bailyn, C.D. 1996, ApJ 459, 226

Rhoades, C.E., Jr., Ruffini, R. 1974, Phys. Rev. Letters 32, 324

Shahbaz, T., Ringwald, F.A., Bunn, J.C., et al. 1994, MNRAS 271, L10

Shahbaz, T., Bandyopadhyay, R., Charles, P.A., Naylor, T. 1996, MNRAS 282, 977

Shahbaz, T., Naylor, T., Charles, P.A. 1997, MNRAS 285, 607

Shahbaz, T., van der Hooft, F., Casares, J., Charles, P.A., van Paradijs, J. 1999a, MNRAS 306, 89 
Shahbaz, T., Bandyopadhyay, R.M., Charles, P.A. 1999b, A\&A 346, 82

Shahbaz, T., Groot, P., Phillips, S.N., Casares, J., Charles, P.A., van Paradijs, J. 2000, MNRAS 314, 747

Tanaka, Y., Lewin, W.H.G. 1995, in: W.H.G. Lewin, J. van Paradijs, E.P.J. van den Heuvel (eds.), X-ray Binaries (Cambridge: CUP), p. 126

Thorsett, S.E., Chakrabarty, D. 1999, ApJ 512, 288

Trimble, V.L., Thorne, K.S. 1969, ApJ 156, 1013

van der Klis, M., Clausen, M., Jensen, K., Tjemkes, S., van Paradijs, J. 1985, A\&A 151, 322

Wagner, R.M., Foltz, C.B., Shahbaz, T., Casares, J., Charles, P.A., Starrfield, S.G., Hewett, P. 2001, ApJ 556, 42

Webb, N.A., Naylor, T., Ioannou, Z., Charles, P.A., Shahbaz, T. 2000, MNRAS 317, 528

Wilson, R.E., Devinney, E.J. 1971, ApJ 166, 605

Zel'dovich, Ya.B., Guseynov, O.H. 1966, ApJ 144, 840

\section{Discussion}

CherepashchuK: Recently Antokhina, Cherepashchuk \& Shalba proposed a new method of determination of parameters of X-ray binaries with black holes based on the variability of absorption line profiles of the optical star with the phase of orbital period. Such variability is due to complicated temperature distribution over the surface of tidally deformed star. What is your feeling about the possibility to realize this method, using new generation telescopes like VLT, Keck, etc.?

Orosz: The method is in principle a good one, but in practice one needs high resolution, high signal-to-noise optical spectra. We have a few VLT-UVES spectra of the brightest system, V4641 Sgr, but ideally we would want spectra over the whole orbit. Apart from V4641 Sgr, it is not feasible to do this for any other X-ray transient.

KOENIGSBERGER: Are the ages of all theses systems (after the SN explosion) actually large enough for them to have circularized and synchronized?

Orosz: The X-ray transients with low mass, Roche lobe filling companions can be quite old, easily $10^{9} \mathrm{yr}$, so there has been ample time for circularization and synchronization. As for Cyg X-1, it's orbit is circular, and we would expect it to be synchronous as well, since in general that that timescale is shorter.

Moffat: I know this is an old question ...: Is the low observed number of $\mathrm{BH}+$ massive companion (O-type) binaries in the Galaxy compatible with the assumption that all stars of $M_{\mathrm{i}}=40 \mathrm{M}_{\odot}$ become BHs, given the complexities of accretion and the possibility of smothered or sleeping systems? What about Cyg X-3?

Orosz: Norbert Langer shared in his presentation that it is very difficult to make relatively massive black holes in close binaries. (Recall that we need close binaries to have an X-ray binary that can be selected.) However, given the small numbers of sources and the uncertainties in the models, we are not prepared to make firm comparisons between theory and observations. Cyg X-3 is certainly a black hole candidate. However, I am not aware of a reliable velocity curve for the companion. That system is difficult to observe owing to the large extinction and the strong wind from the companion. 